\title{
Coulisses
}

Revue de théâtre

42 | Printemps 2011

Racine : Théâtre et émotion

\section{Bérénice de Racine, la belle étrangère du théâtre de Gwenaël Morin}

Catherine Ailloud-Nicolas

\section{(2) OpenEdition}

1 Journals

Édition électronique

URL : https://journals.openedition.org/coulisses/592

DOI : 10.4000/coulisses.592

ISSN : 2546-9460

Éditeur

Presses universitaires de Franche-Comté

Édition imprimée

Date de publication : 15 février 2011

Pagination : 81-89

ISBN : 978-2-84867-316-5

ISSN : $1150-594 X$

Référence électronique

Catherine Ailloud-Nicolas, «Bérénice de Racine, la belle étrangère du théâtre de Gwenaël Morin », Coulisses [En ligne], 42 | Printemps 2011, mis en ligne le 30 novembre 2016, consulté le 29 décembre 2022. URL : http://journals.openedition.org/coulisses/592; DOI : https://doi.org/10.4000/coulisses. 592

Tous droits réservés 


\title{
Bérénice de Racine, la belle étrangère du théâtre de Gwenaël Morin
}

\author{
CATHERINE Ailloud-NiCOLAS, \\ Université Claude-Bernard - Lyon I
}

Dans la majeure partie des cas, la mise en scène d'un texte classique s'inscrit dans des chronologies emboîtées. Elle s'envisage comme la rencontre particulière d'un artiste avec un texte à un moment donné. Elle constitue une étape plus ou moins importante dans la série des spectacles qu'il réalise, dans ce que l'on pourrait nommer son œuvre spectaculaire. Et lorsque le résultat est particulièrement réussi, la mise en scène prend un statut de référence et entre ainsi dans l'histoire des mises en scène de la pièce ${ }^{1}$ voire dans l'histoire de la mise en scène tout court.

Cette façon d'envisager la mise en scène est profondément remise en cause par la façon de travailler de Gwenaël Morin. En effet, dans le projet de ce jeune metteur en scène s'affiche non pas le désir de céder à la logique de succession de spectacles et de s'inscrire dans un temps individuel et collectif plus ou moins contraint, mais l'affirmation d'une approche synchronique à savoir d'une cohérence profonde entre des principes qu'il a énoncés dans un manifeste, une méthodologie d'organisation du travail et une esthétique. Il y a, dès lors, un renversement de perspective puisque la mise en scène du texte particulier se trouve au service d'une démarche globale qui la dépasse, l'englobe et agit fortement sur elle. On a alors le sentiment que les contraintes qui s'imposent ou sont imposées au texte de l'extérieur sont dominantes par rapport à la stricte prise en compte de l'œuvre. La question est de savoir comment cela influe sur la lecture de la pièce.

1. Certaines pièces se prêtent plus que d'autres à cela. Ainsi, il est possible de faire une histoire des mises en scène de Dom Juan ou de Tartuffe. Cela tient à un double phénomène : les mises en scène retenues ont apporté un nouvel éclairage sur la pièce et en même temps ont constitué des jalons dans l'histoire même de la mise en scène. Bérénice échappe à ce phénomène. Pourquoi ? Est-ce parce qu'elle apparait comme fermée, univoque? 
Il convient donc pour appréhender la mise en scène de Bérénice par Gwenaël Morin, de comprendre la pensée générale de ce metteur en scène, sa façon d'envisager le théâtre, la démarche qui en découle. Nous ferons une rapide analyse de ces aspects avant de laisser la parole à Gwenaël Morin qui nous a accordé un entretien.

\section{Une démarche au service d'un engagement}

En juin 2008, Gwenaël Morin conçoit un texte, le discours de Turin², lu en italien puis traduit en français, dans lequel il affirme certains principes, et insiste en particulier sur sa conception particulière du rapport au temps et en particulier sur son goût pour un thêâtre de l'urgence: "Je ne vis pas l'urgence comme une crise, je l'utilise pour produire un théâtre de l'excès et de l'exagération ». Loin d'être une posture théorique, cette affirmation trouve sa réalité dans une modalité de travail : «Je veux faire sortir le théâtre du paradis de l'exceptionnel avec le théâtre permanent. Je veux qu'il ait lieu tous les jours. Je veux expérimenter la beauté du théâtre dans la vie quotidienne. Je veux faire du théâtre permanent».

Il ne s'agit pas là d'une simple affirmation de principe puisqu'elle a trouvé corps dans une expérience qui s'est déroulée pendant l'année 2009 à Aubervilliers. Une équipe de comédiens ${ }^{3}$, rassemblée par Gwenaël Morin, décida de faire du théâtre tous les jours ce qui signifiait inventer un rythme de travail particulier. Le site des laboratoires d'Aubervilliers en résume les trois règles: «jouer tous les jours» sauf les dimanches et les lundis, "répéter tous les jours », «transmettre en continu $»^{4}$. Ces modalités renversent évidemment en profondeur la façon de faire du théâtre. Celui-ci devient un métier, une activité à part entière. Il ne s'agit plus d'organiser le temps en fonction de la création avec ses étapes bien connues : répétition, production, diffusion dans une linéarité et une succession éprouvées à chaque projet. Il s'agit, en mettant le théâtre au centre, de nier une organisation temporelle régie principalement par des principes économiques. Le projet de la troupe, celui de faire du théâtre ensemble, est plus important que le projet singulier organisé, celui de «monter une pièce ». Le temps de la troupe s'oppose au temps éphémère de l'équipe de création rassemblée pour une mise en scène.

Le théâtre permanent pose alors la question du répertoire ad hoc pour convenir à cette organisation. Or, Gwenaël Morin s'est lancé dans un projet ambitieux qu'il a appelé le "théâtre de la responsabilité ». Il s'agit de monter

2. Consultable sur le site : archives.leslaboratoires.org/content/view/367/lang,fr.

3. Julian Eggerickx, Barbara Jung, Grégoire Monsaingeon.

4. «Chaque matin, lors d'ateliers ouverts à tous, un acteur de la troupe a la charge de transmettre individuellement le rôle qu'il assume chaque soir en représentation. » 
des pièces dont le titre est le nom du personnage principal. S'ensuit donc de janvier à décembre 2009 une série de spectacles présentés comme des adaptations ${ }^{5}$. Bérénice d'après Bérénice de Racine s'inscrit donc dans un ensemble et la pièce existe autant en soi qu'à l'intérieur de cet ensemble où elle rejoint Antigone d'après Antigone de Sophocle comme pièce dont le titre est un prénom féminin, ou bien Tartuffe d'après Tartuffe de Molière comme pièce du $17^{\mathrm{e}}$ siècle.

La particularité de la pièce en revanche c'est qu'elle est la seule à être présente dans le discours de Turin dans une section intitulée : "Langage/Bérénice ». Elle sert à définir ce que Gwenaël Morin appelle le «syndrome de Bérénice ou la tragédie du langage » et qu'il décrit de la façon suivante :

Bérénice porte le langage comme l'enfant qu'elle n'aura jamais avec Titus. Nous naissons et vivons séparés

Entre vous et moi il y a le monde

Le langage essaie de transformer le monde entre nous

Pour le faire devenir notre enfant

Le nom de notre enfant est théâtre.

Il y a là, à la fois une réflexion dramaturgique sur Bérénice et l'utilisation du personnage comme emblème d'une problématique qui traverse le théâtre de Gwenaël Morin. La pièce de Racine se trouve donc à la confluence de la réflexion et de la production du metteur en scène, place centrale qu'il convient de souligner. Néanmoins, on peut se demander si elle est si naturellement que cela intégrable dans son esthétique.

\section{Un projet, une esthétique, un univers}

Les derniers spectacles de Gwenaël Morin font apparaittre des lignes de force, des axes de recherche suffisamment nets pour qu'ils puissent être nommés comme tels, indépendamment du projet qui consiste à vouloir monter et montrer des pièces centrées sur un personnage éponyme. Au-delà de cette question du personnage qui donne son nom à sa pièce, se met en place une réflexion sur le théâtre qui prend la forme d'un feuilletage cohérent, à l'œuvre dans les divers spectacles à des degrés divers.

On peut considérer qu'il y a un premier niveau qui est celui de l'interprétation de la pièce, niveau qui met en place une dramaturgie du sens et de la contrainte. Du sens parce que le lien avec aujourd'hui est toujours posé, plus ou moins discrètement. De la contrainte, parce que l'inadéquation pratiquement systéma-

5. Il y avait eu avant cela Wilhelm Tell d'après Guillaume Tell de Schiller, Philoctète d'après Philoctète de Sophocle. Par ailleurs, l'idée de la permanence s'était déjà exprimée dans une expérience organisée au théâtre de L'Élysée, à Lyon. Pendant un mois, ce lieu a accueilli des lectures à voix haute, effectuées par le public, en continu. Tous les soirs, un chœur de spectateurs sortait du théâtre pour aller déclamer une phrase d'un philosophe, choisie par la Compagnie. 
tique entre le nombre d'acteurs et le nombre de personnages induit par là même des transformations, des choix de montage ou de résolution scénique. La pièce se trouve interrogée de l'intérieur mais elle est aussi questionnée à partir de contraintes qui la dépassent. Dans le théâtre de Gwenaël Morin, dramaturgie externe et interne cohabitent et dialoguent en permanence. Ainsi, en ce qui concerne Bérénice, la note d'intention est à ce niveau exemplaire. Elle montre une interprétation de la pièce claire et précise : "Titus a aimé et n'aime plus Bérénice, ils vont devoir se séparer. Ce n'est pas un renoncement, ce n'est pas une décision, c'est un arrêt» puis «Bérénice fait l'expérience déchirante de cette contradiction tragique : voulant retenir Titus elle le perd, entre Titus et elle, règnent désormais les mots, le langage, le monde, les hommes. L'amour chassé va mourir. Je veux, par le biais des protagonistes de la pièce de Racine, mettre en scène une lutte à mort entre l'amour et la parole ». Cette interprétation s'ouvre sur une problématique plus large, qui dépasse l'histoire racontée : «Je veux faire Bérénice d'après Bérénice de Racine pour dire et montrer comment la parole sépare. Là où l'amour, dans la passion, voudrait abolir le monde qui sépare les amants, la parole le rétablit inexorablement comme condition même de sa manifestation. Là où l'amour voudrait fondre et unir les amants, la parole coupe, sépare, éloigne, creuse l'espace, réhabilite le monde ». Ces deux réflexions sur le sens permettent de comprendre comment, interrogeant ponctuellement une pièce, une mise en scène finit par interroger le monde et le théâtre, par dire quelque chose d'aujourd'hui qui dépasse le propos initial ou même ce que l'on veut montrer d'une pièce. Il y a là de façon exemplaire le double niveau de sens que fait émerger une dramaturgie interne réussie. Mais, la pièce étant jouée par quatre acteurs, elle est aussi l'objet des effets de la dramaturgie externe. Car le choix d'une équipe plus restreinte de comédiens va entraîner condensations, coupes, restrictions qui font que Bérénice se trouve resserrée, densifiée, recentrée autour d'un noyau dur.

Ce premier niveau, celui de l'interprétation de la pièce, qui fait qu'on ne perd jamais le fil de ce qui est raconté et en même temps de ce que l'on veut nous raconter, se fait dans le cadre d'une esthétique forte de théâtre en train de se faire, de théâtre pauvre. L'allusion à l'éphémère est constante : carton, papiers, bouts de ficelle s'affichent sur la froideur du rideau de fer ou sur les murs nus du théâtre. Dans Bérénice, comme dans de nombreux spectacles du Théâtre de la Responsabilité, le texte est collé sur la scénographie, le carton domine, une carte de la Palestine fait face à une photo d'un parlement, une petite scène sert de lien entre deux espaces de non jeu. Urgence et éphémère, comme une marque de fabrique de ce théâtre mais remotivée ici par l'essentielle simplicité de la pièce.

Le deuxième niveau est celui de la rupture. Ce niveau est très repérable en termes de jeu. Le texte se fait entendre dans une esthétique de jeu qui affirme une très forte théâtralité : adresse directe au public qui n'est jamais oublié par l'acteur, voix portée jusqu'au cri, rôles masculins joués par des femmes, convention assumée qui ne cherche pas l'illusion, équation entre le silence et le noir. L'acteur 
circule dans un va-et-vient entre incarnation et témoignage. De temps en temps, il plonge dans la fable, il plonge dans le théâtre. Puis il en sort pour être dans le cri, dans l'adresse, dans l'interpellation. Rupture dans le type de jeu. On passe du théâtre à la conversation quotidienne, par des changements de niveaux de voix, par l'irruption d'une connivence déconnectée de la situation. Rupture aussi entre le personnage et l'acteur, à des moments où, quittant son personnage, l'acteur s'affirme comme tel, désigne ce qui est en train de se passer, rendant tout à coup le présent incroyablement tangible. C'est une interruption de la pièce, de la fable, et en même temps tout est parfaitement intégré au spectacle. Le spectacle des personnages laisse la place au spectacle des acteurs. Le théâtre laisse la place à la performance. Et il n'est pas rare alors, qu'un des acteurs devienne spectateur à la fois de ce qui se passe sur scène et du public. Dans la mise en scène de Bérénice, on retrouve quelques uns de ces aspects, mais atténués. Certes, les acteurs ne sont jamais cachés et attendent patiemment de devenir les personnages. Certes, Julian Eggerickx s'installe au milieu des spectateurs, attendant de sauter sur l'estrade pour interpréter Antiochus. Mais la frontière entre jeu et performance est dans cette mise en scène plus rarement franchie que dans d'autres de la série, comme si Bérénice résistait, comme si elle refusait de se prêter à un autre mode que celui de l'incarnation.

Le troisième niveau est celui de la mise en danger du théâtre par le théâtre. Mise en danger de l'acteur qui doit, selon les spectacles, descendre les travées dans le noir, qui doit recevoir des pavés, qui doit hurler à en perdre la voix. Théâtre de la fatigue et de l'épuisement. Mise en danger de la représentation par le refus de l'efficacité, par la mise en place de ce qui est susceptible de mal tourner. Mise en danger enfin par le dérapage scénique, par ces moments où l'on a l'impression d'un théâtre de la violence extrême, du n'importe quoi organisé, de l'excès. Le théâtre déborde alors comme du lait sur le feu avant de reprendre sagement son cours. Là encore Bérénice fait figure d'étrangère dans l'univers de Gwenaël Morin. Seuls deux procédés rappellent la nécessité de la fracture. Tout d'abord, chaque " hélas » est ponctué par un gong. Ce rituel, qui crée de l'étrangeté et de l'insolite, finit par devenir un outil dramaturgique car il souligne la présence obsédante du mot dans l'œuvre. C'est une ponctuation musicale qui devient une ponctuation de sens. Enfin, tout à coup, tous les acteurs s'interrompent pour chanter ensemble les deux premières phrases de la chanson: «Words ». La répétition en boucle crée un effet comique comme la réitération du gong sur le mot «hélas ». C'est un dérapage par rapport à la pièce mais qui ne prend pas la forme presque hystérique des dérapages dans d'autres mises en scène de Gwenaël Morin. On est ici plus du côté de l'étrangeté, de l'incongruité.

Ces trois niveaux, ce feuilletage mettent au premier plan obsessionnellement, obstinément, les questions essentielles : pourquoi et comment fait-on du théâtre?

Et c'est à partir de ces questions que j'ai interrogé Gwenaël Morin. 


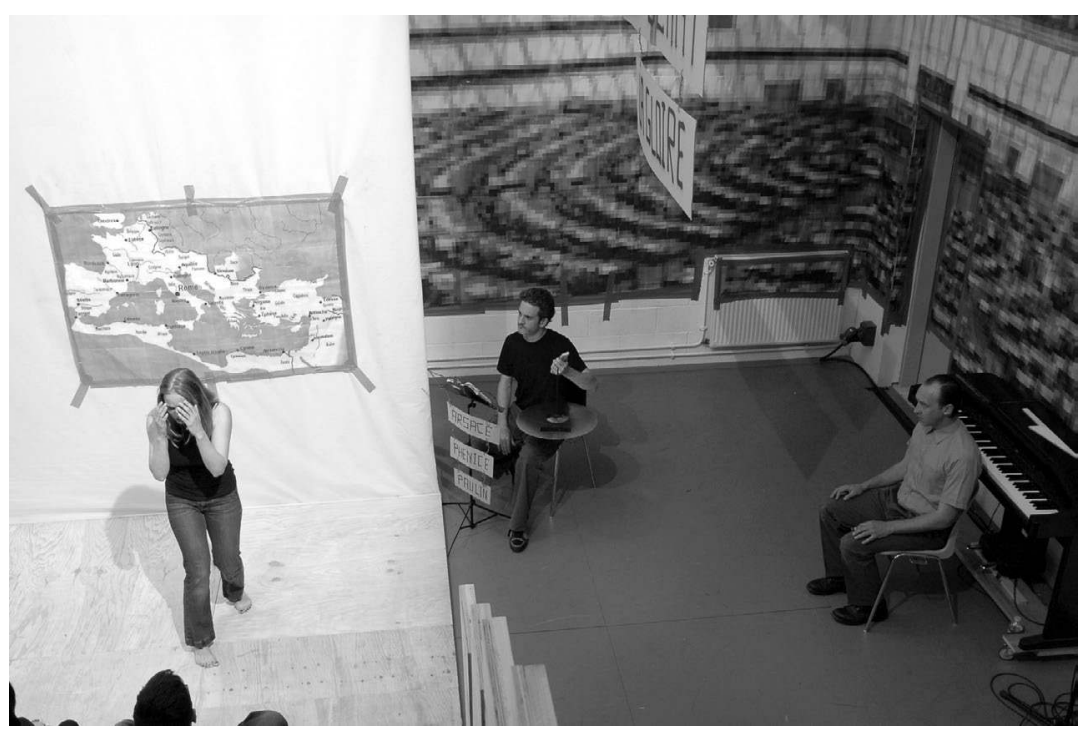

Photo : Julie Pagnier

\section{Parole d'artiste}

Comment s'est opéré le choix des textes du Théatre de la responsabilité?

J'ai voulu constituer un corpus de textes en choisissant parmi les plus connus du répertoire occidental, et j'ai réduit le spectre en m'intéressant, parmi eux, uniquement aux textes libres de droit dont le titre est le nom d'un personnage. Avant de parler d'un projet dramaturgique, je voulais d'abord revendiquer un programme bâti sur des règles élémentaires et partageables par tous, ma volonté étant d'affirmer «le théâtre » le plus simplement possible et le plus radicalement possible au cœur de la cité. Clamer Hamlet au cœur de la ville est pour moi une exhortation au théâtre. Le programme du théâtre permanent était constitué dans l'ordre par : Lorenzaccio d'après Lorenzaccio de Musset, Tartuffe d'après Tartuffe de Molière, Bérénice d'après Bérénice de Racine, Antigone d'après Antigone de Sophocle, Hamlet d'après Hamlet de Shakespeare et Woyzeck d'après Woyzeck. de Büchner.

\section{Pourquoi n'as-tu choisi que des classiques?}

Je voulais que le théâtre permanent soit le plus inclusif possible. J'ai pris encore une fois la décision de ne choisir que des textes appartenant au domaine 
public donc à nous tous, des chefs-d'œuvre qui soient des lieux communs de notre culture occidentale. Le théâtre n'est-il pas un lieu commun, un lieu où faire une certaine expérience du commun? Monter des textes connus et appartenant au domaine public m'a conduit mécaniquement vers les grand classiques. L'expérience aidant, je peux dire aussi qu'avec un chef-d'œuvre classique, la question de la légitimité du texte ne se pose plus. Dès lors, la question n'est pas pourquoi faire par exemple Tartuffe, mais comment faire Tartuffe, encore et encore, c'est-à-dire comment faire du théâtre ? Paradoxalement, monter ces vieux textes est une façon d'interroger, aujourd'hui, une certaine nécessité du fait théâtral.

L'aventure du Théatre Permanent telle que tu l'as conduite au Laboratoire d'Aubervilliers est achevée, comment se prolonge-t-elle? Les mises en scène continuent-elles à tourner?

Le Théâtre Permanent est devenu aujourd'hui la troupe du Théâtre Permanent. Autour de ce travail, on s'est constitué un répertoire, un programme, et nous avons envie de voir comment les différents lieux qui nous sollicitent, arrivent à aborder cette manière de travailler différents spectacles dans le cadre d'un répertoire. Maintenant la troupe est devenue un réel outil, qui ne demande qu'à dialoguer avec les autres théâtres dans le but de les investir, de les faire avancer. Ce n'est donc pas du tout un théâtre fermé, car cela nous permet de pouvoir sans arrêt revoir le rapport au temps, de transformer la pièce, etc. Le devenir du Thêâtre Permanent est vraiment la troupe du Théâtre Permanent avec qui, d'ailleurs, je n'ai pas du tout décidé d'une date de fin.

La troupe s'est constituée autour du travail et non l'inverse. Nul ne savait réellement où il mettait les pieds, mais étant donné qu'il ne s'agit que d'acteurs avec qui j'avais déjà travaillé dans le passé, ils pouvaient avoir une vague idée de ce à quoi s'attendre. On avait décidé de la durée d'un an dans un même lieu, avec une équipe stable, autour d'un programme clair mais sans pour autant décider d'une troupe fixe, qui comme le répertoire choisi, est apparue ensuite comme une évidence.

\section{Quelle est la place de Bérénice dans ce projet? Comment as-tu abordé la pièce?}

L'abstraction de l'écriture de Racine a été d'une approche plus simple pour moi que ne l'a été celle de l'écriture à double fond de Molière et du Tartuffe que nous venions de traverser. Nous avons beaucoup travaillé à la table, ce qui était nouveau pour moi. Durant de longues périodes, j'ai écouté le texte, sans velléité de vouloir être productif tout de suite. À l'intuition, je demandais aux acteurs de modifier tel rythme, telle intensité... La clarté absolue de la langue de Racine nous a permis de nous concentrer presque exclusivement sur la forme. Une fois la forme établie, le sens éclatait. 


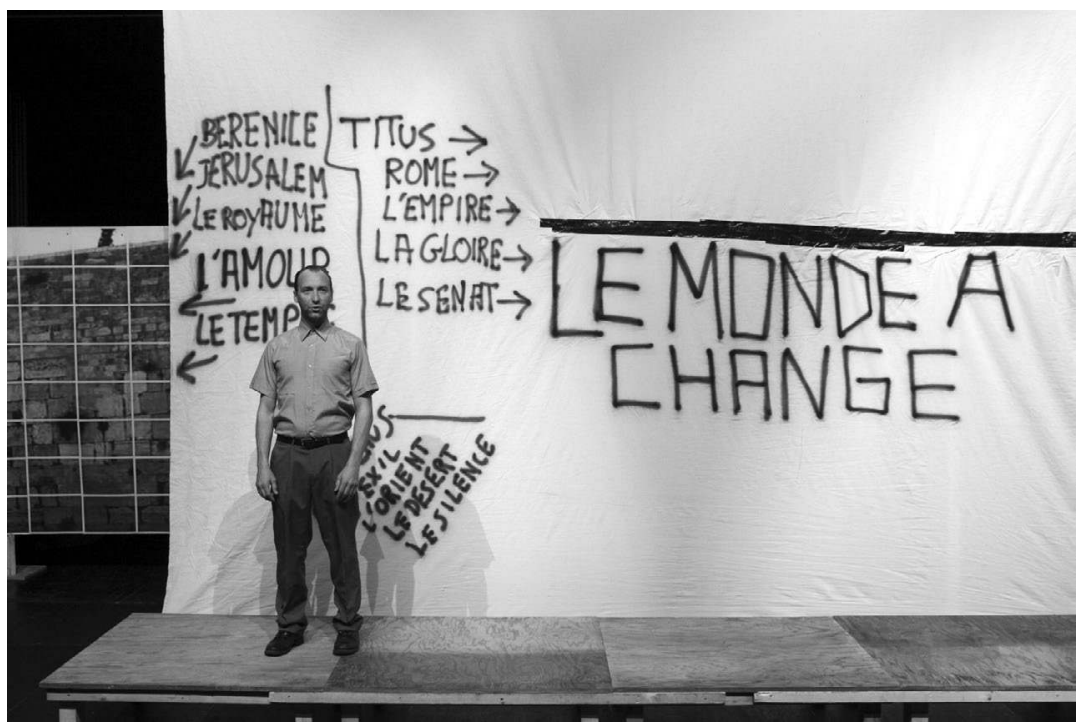

Photo : Élodie Laimene-Erard

Grégoire Monsaingeon, qui interprète Titus, a fait sur le texte un travail d'adaptation radical qui a consisté à dépouiller la pièce d'une énorme part des ses références à l'histoire romaine. Nous voulions nous concentrer sur la relation entre les trois personnages: Titus, Bérénice et Antiochus. Un quatrième acteur lit la partition des confidents respectifs des trois protagonistes principaux. La pièce dure un peu plus d'une heure interrompue par un intermède musical (Words de Fr David et Da da da de Trio).

La mise en scène est en second. Tout se joue sur une bande d'un mètre de large face public sans que jamais les acteurs se croisent. Pas de costume, plein feu du début à la fin. Le décor : une image du Mur des lamentations à jardin, le côté de Bérénice, Reine de Palestine. Une image de l'hémicycle de l'assemblée européenne à cour, le côté de Titus, Empereur de Rome. Antiochus se tient dans les escaliers du côté du public, il va et vient sur la scène.

\section{Comment s'est fait le travail avec les acteurs?}

Il n'y a pas d'ironie chez Racine, pas de distance. C'est d'une grande clarté. Une fois accepté le formalisme de la versification, tout est simple, ce qui ne veut pas dire facile. Tout le travail avec les acteurs a été de réaliser ensemble cette simplicité. Pour me rendre sensible à la simplicité, j'essaye de ne pas 
m'intéresser à ce qui est dit, mais plutôt de produire des formes qui finalement me révèlent le sens. Je suis souvent le dernier de l'équipe à comprendre ce qui est en jeu dans une scène, uniquement parce que je ne veux pas écouter ce que je n'entends pas. Parfois un simple mot, un accident de plateau ou un malentendu insignifiant font surgir le sens... Je ferme souvent les yeux quand je mets en scène, je me rends aveugle... Je ne veux pas mettre en scène ce que j'ai compris, je veux mettre en scène pour comprendre. Le travail avec les acteurs ressemble donc à une danse d'abord abstraite, arbitraire et hésitante de laquelle se dégagent progressivement, par la répétition, des évidences élémentaires.

\section{Comment prépares-tu le travail, lis-tu des essais?}

Je lis plutôt pendant le travail qu'avant le travail. Je préfère me retrouver dans la situation de devoir faire au jour le jour. Tout ce qui est idéologique, méthodique a tendance à me poser problème, car lorsqu'on élabore une méthode, on adopte une attitude un peu conservatrice : on produit ces méthodes, ces idéologies sur l'expérience qu'on a déjà du monde. J'essaye, en toute modestie, de rester au maximum disponible à ce qui va arriver.

\section{Pourquoi le texte est-il présent, collé sur les murs, sur ta scénographie?}

J'ai décidé depuis Mlle Julie, de toujours exposer sur scène, d'une manière ou d'une autre, le texte imprimé et jusqu'à aujourd'hui, je me suis tenu à cette règle arbitraire. Au début de la répétition d'un nouveau texte, $j$ 'ai pris l'habitude de le placarder au mur, dans l'espace de jeu. C'est une sorte de rituel, de base de travail. Le texte dans l'espace, le travail de mise en scène peut commencer.

\section{Peux-tu faire un bilan de cette expérience?}

Non, faire du théâtre n'est pas une façon pour moi de remplir des objectifs, et je ne sais pas regarder en arrière. Je ne peux que réaffirmer ici ma volonté de faire et de continuer à faire du théâtre. Concernant Bérénice, je sais déjà que de cette première exposition, de ce premier précipité, naîtra la nécessité de visiter encore et encore ce texte et que je le mettrai en scène à nouveau dans quelque temps, peut-être d'ailleurs pour refaire la même chose. Le théâtre, je crois, a cette vocation-là : épuiser les possibilités de la parole pour donner à voir ce qu'elle ne saurait contenir. Répéter, répéter sans cesse avec ténacité et insistance jusqu'à ce que quelque chose apparaisse. 